\title{
QRS Loop Folding Phenomenon in Vectorcardiogram of Healthy Individuals
}

\author{
Golriz Sedaghat $^{1,2}$, Muammar M Kabir ${ }^{1}$, Larisa G Tereshchenko ${ }^{1}$ \\ ${ }^{1}$ Knight Cardiovascular Institute, Oregon Health \& Science University, Portland, OR, USA \\ ${ }^{2}$ Portland State University, Portland, OR, USA
}

\begin{abstract}
Recently, we developed a novel approach to study the rapid and sudden changes in the direction of ventricular activation called folding phenomenon. In order to better understand this phenomenon, we were interested in studying the variation of the orientation of $\overrightarrow{Q R S_{l}}$ for $i=1 \ldots N$ where $\overrightarrow{Q R S_{l}}$ represents the $Q R S$ vector at the $i^{\text {th }}$ sample point. In this study, we described the orientation of the $\overrightarrow{Q R S}$ vector and the rotation of its frame of reference in details in 81 healthy participants from the Intercity Digital Electrocardiogram Alliance (IDEAL) study using unit quaternions and Euler angles. We observed that the variance of the elevation of rotation axis $\vec{u}$ was

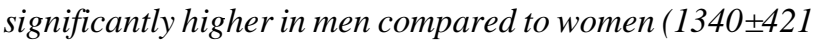
vs. $1063 \pm 381, P=0.003$, respectively). Additionally, there was a significant negative correlation between the variance of the azimuth of $\vec{u}$ and height (CC $=-0.26$, $P=0.019)$ while the elevation of $\vec{u}$ and height were significantly positively correlated $(C C=0.24, P=0.034)$. Moreover, the elevation of $\vec{u}$ had a positive significant correlation with weight and body surface area $(C C=0.22$, $P=0.045$ and $C C=0.26, P=0.020$ respectively).
\end{abstract}

\section{Introduction}

Vectorcardiogram (VCG) demonstrates the projection of the instantaneous changes in the direction and magnitude of the electrical activity of the heart on the coronal (frontal), transverse (horizontal) and sagittal (vertical) planes [1]. Recently, we studied the VCG morphological parameters of QRS loop including roundness, planarity, thickness, rotational and dihedral angles [2]. We showed that patients with infarctcardiomyopathy and sustained monomorphic ventricular tachycardia (VT) had lower planarity index meaning that the movement of electrical activity in their hearts depicted a more complex trajectory. Furthermore, it was observed that VT subjects had significantly more variable dihedral angle which indicated abrupt changes in the QRS loop main plane [2]. Consequently, a new qualitative feature of abnormal QRS loop, called "folding” phenomenon was introduced as a predominant characteristic of QRS loop in patients with myocardial infarction and ventricular tachycardia substrate [1,2]. However, it is important to understand the manifestation of folding phenomenon in healthy individuals to determine their manifestation in clinical settings. Therefore, in this paper we focused merely on studying the variation of orientation of the QRS vector and its frame of reference in the QRS loop of healthy individuals using quaternions and Euler angles.

\section{Methods}

\subsection{Subjects}

For this study 81 healthy individuals from the Intercity Digital Electrocardiogram Alliance (IDEAL) study, funded by NIH The Telemetric and Holter ECG Warehouse (THEW) initiative [3], who had sinus ECG and no history of any chronic disease were included.

The orthogonal Frank ECG signal was recorded at 1000 $\mathrm{Hz}$ using the SpaceLab-Burdick digital Holter recorder. Information on age, gender, race, height, weight, systolic and diastolic blood pressure and smoking status as well as Body Surface Area (BSA) were reported elsewhere [4].

MATLAB customized (MathWorks, Inc, Natick, MA) software was developed for automated ECG and VCG analysis. Respiration was removed using shifting, rotating and rescaling VCG loops [4]. Five clean consecutive sinus beats were selected and their median beat was constructed in order to measure the VCG parameters explained in the following sections

\subsection{Spatial orientation}

In geometry, orientation of an object describes its position relative to a defined frame of reference. Rotation matrices, Euler angles and unit quaternions are three widely used methods describing the orientation of a rigid body in three dimensions. Employing each method may widely vary based on the application. In this study we used unit quaternions to describe the orientation of QRS vectors since they are more efficient and compact representation of orientation compared to rotation matrices as well as they 
avoid the gimbal lock problem of Euler angles [5]. Furthermore, Euler angles were employed to measure the orientation angles between the frames of reference of QRS vectors.

\subsection{Quaternions}

Quaternions are considered as hyper-complex numbers of rank 3, $4 \ldots \mathrm{n}$. A quaternion is a 4-tuple element in $R^{4}$.

$$
q=\left(q_{0}, q_{1}, q_{2}, q_{3}\right)
$$

where $q_{0}, q_{1}, q_{2}, q_{3}$ are real numbers. $q_{0}$ is called the scalar part while $\vec{q}=\left(q_{1}, q_{2}, q_{3}\right)$ is the vector part of the quaternion. Therefore, a quaternion can also be defined as the sum of a scalar and a vector and be presented as:

$$
q=q_{0}+\vec{q}=q_{0}+\vec{\imath} q_{1}+\vec{\jmath} q_{2}+\vec{k} q_{3}
$$

where $\vec{\imath}, \vec{\jmath}$ and $\vec{k}$ are the standard orthonormal basis in $R^{4}[5,6]$.

\subsection{Quaternion rotation operators}

Unit quaternions are defined as quaternions with a magnitude of one and are commonly used to represent a rotation with an angle of $\alpha$ about an axis $\vec{u}$. A unit quaternion can be demonstrated in axis-angle form as [5]:

$$
q(\alpha, \vec{u}) \triangleq\left(\begin{array}{c}
\cos \left(\frac{1}{2} \alpha\right) \\
\vec{u} \sin \left(\frac{1}{2} \alpha\right)
\end{array}\right)
$$

Using unit quaternions, a rotation operator, $L_{q}$ on $\vec{v}$ can be defined as [6]:

$$
\vec{w}=L_{q}(\vec{v})=q \vec{v} \bar{q}
$$$$
q \vec{v} \bar{q}=\left(q_{0}^{2}-|\vec{q}|^{2}\right) \vec{v}+2(\vec{q} \cdot \vec{v}) \vec{q}+2 q_{0}(\vec{q} \times \vec{v})
$$

where $\bar{q}$ is the conjugate of the quaternion $q$ and is calculated as following:

$$
\bar{q}=q_{0}-\vec{q}=q_{0}-\vec{\imath} q_{1}-\vec{\jmath} q_{2}-\vec{k} q_{3}
$$

$|\vec{q}|$ is the norm of the vector part of the quaternion $q$ :

$$
|\vec{q}|=\sqrt{q_{1}^{2}+q_{2}^{2}+q_{3}^{2}}
$$

$\vec{q} \cdot \vec{v}$ and $\vec{q} \times \vec{v}$ are respectively the dot product and cross product of vectors $\vec{q}$ and $\vec{v}$.

The operator $L_{q}$ can be geometrically interpreted as a rotation of the vector $\vec{v}$ through an angle $\alpha$ about the axis $\vec{q}$ or $\vec{u}[6]$.

\subsection{Spatial $\overrightarrow{Q R S}$ vectors}

The origin of the QRS loop was chosen as halfway between the two points that were closest in space and furthest in time [4]. Spatial QRS vectors, $\overrightarrow{Q R S_{l}}$ were obtained by joining the QRS loop origin to each sample point of the loop.

\subsection{Orientation of $\overrightarrow{Q R S}$ vectors}

The changes of the orientation of $\overrightarrow{Q R S_{l}}$ over time was calculated by the rotation unit quaternion $q_{i}$ which describes the rotation of the $\overrightarrow{Q R S}$ from time $i$ to $i+1$.

For this study, since the sequence of $\overrightarrow{Q R S_{l}}$ vectors were known, we had to determine the rotation unit quaternion sequence, $q_{i}$. Although there are infinitely many rotations that would rotate $\overrightarrow{Q R S_{l}}$ in the direction of $\overrightarrow{Q R S_{l+1}}$, we were interested in the rotation unit quaternion that would rotate the two consecutive $\overrightarrow{Q R S}$ vectors within the shortest arc. The angle of rotation $\alpha_{i}$ as well as the axis of rotation $\overrightarrow{u_{\imath}}$ were calculated as following:

$$
\begin{aligned}
& \alpha_{i}=\tan ^{-1}\left(\frac{\left|\overrightarrow{Q R S_{l}} \times \overrightarrow{Q R S_{l+1}}\right|}{\overrightarrow{Q R S_{l}} \cdot \overrightarrow{Q R S_{l+1}}}\right) \\
& \overrightarrow{u_{\imath}}=\frac{\overrightarrow{Q R S_{l}} \times \overrightarrow{Q R S_{l+1}}}{\left|\overrightarrow{Q R S_{l}} \times \overrightarrow{Q R S_{l+1}}\right|}
\end{aligned}
$$

Acquiring $\alpha_{i}$ and $\overrightarrow{u_{l}}$, the rotation quaternion sequence $q_{i}$ was calculated using equation (3). The azimuth (AzR) and the elevation (EIR) of the axis of rotation $\overrightarrow{u_{\imath}}$ was calculated by converting the Cartesian coordinates into polar coordinates as described previously [2]:

$$
\begin{aligned}
& \text { azimuth }=\tan ^{-1}\left(\frac{\overrightarrow{u_{l_{y}}}}{\overrightarrow{u_{l_{x}}}}\right) \\
& \text { elevation }=\tan ^{-1}\left(\frac{{\overrightarrow{u_{l_{z}}}}^{2}}{\sqrt{{\overrightarrow{u_{l_{x}}}}^{2}+{\overrightarrow{u_{l}}}^{2}}}\right)
\end{aligned}
$$

\subsection{Angular velocity}

In order to calculate the orientation angles, quaternions were converted to Euler angles. Euler angles describe the orientation of one frame of reference relative to another while the object can be assumed stationary. Azimuth $(\psi$ rotation about the $\mathrm{z}$-axis), elevation ( $\theta$ rotation about the $\mathrm{y}$-axis) and tilt ( $\phi$ rotation about the $\mathrm{x}$-axis) angles were calculated as follows:

$$
\begin{aligned}
\phi & =\tan ^{-1}\left(\frac{2 q_{0} q_{1}+2 q_{2} q_{3}}{q_{3}^{2}-q_{2}^{2}-q_{1}^{2}+q_{0}^{2}}\right) \\
\theta & =\sin ^{-1}\left(2 q_{0} q_{2}-2 q_{1} q_{3}\right) \\
\psi & =\tan ^{-1}\left(\frac{2 q_{1} q_{2}+2 q_{0} q_{3}}{q_{3}^{2}+q_{2}^{2}-q_{1}^{2}-q_{0}^{2}}\right)
\end{aligned}
$$

The three Euler angles were used to compute the angular velocity of the $\overrightarrow{Q R S}$ vector across each of the $\mathrm{x}, \mathrm{y}$ and $\mathrm{z}$ axes using the following equations $[7,8]$ :

$$
\begin{aligned}
& \omega_{x}=\dot{\phi} \sin (\theta) \sin (\psi)+\dot{\theta} \cos (\psi) \\
& \omega_{y}=\dot{\phi} \sin (\theta) \cos (\psi)-\dot{\theta} \sin (\psi) \\
& \omega_{z}=\dot{\phi} \cos (\theta)+\dot{\psi}
\end{aligned}
$$

\section{Results}

The study cohort for this study consisted of 38 women and 43 men. The summary of the demographics of the total 
population is reported in table 1.

Table 1. Demographics of the 81 healthy subjects

\begin{tabular}{ll}
\hline Measurement & Healthy subjects (N=81) \\
\hline Age (SD), y & $38(15)$ \\
Body Mass Index (SD), $\mathrm{kg} / \mathrm{m}^{2}$ & $24.5(5.1)$ \\
Height (SD), cm & $170(10)$ \\
Weight (SD), kg & $71.3(16.0)$ \\
Body Surface Area (SD), $\mathrm{m}^{2}$ & $1.82(0.24)$ \\
\hline
\end{tabular}

Table 2. Comparison of orientation of $\overrightarrow{Q R S}$ vector between male and female

\begin{tabular}{lccc}
\hline Measurement & $\begin{array}{c}\text { Female } \\
(\mathrm{N}=38)\end{array}$ & Male (N=43) & $\begin{array}{c}\mathrm{P} \\
\text { value }\end{array}$ \\
\hline Mean $\omega_{x}(\mathrm{SD}), \mathrm{rad} / \mathrm{s}^{*} 10^{4}$ & $6(45)$ & $4(45)$ & 0.845 \\
Mean $\omega_{y}(\mathrm{SD}), \mathrm{rad} / \mathrm{s}^{*} 10^{4}$ & $23(44)$ & $26(116)$ & 0.855 \\
Mean $\omega_{z}(\mathrm{SD}), \mathrm{rad} / \mathrm{s}^{*} 10^{4}$ & $8(33)$ & $-5(43)$ & 0.131 \\
$\begin{array}{l}\text { Mean of azimuth of } \vec{u}, \\
\text { degrees }\end{array}$ & $3.8(19.4)$ & $2.8(20.2)$ & 0.820 \\
$\begin{array}{l}\text { Variance of azimuth of } \vec{u} \\
\text { (SD), degrees }\end{array}$ & $8160(2531)$ & $7257(1533)$ & 0.061 \\
$\begin{array}{l}\text { Mean of elevation of } \vec{u}, \\
\text { degrees }\end{array}$ & $100.3(7.9)$ & $102.9(8.1)$ & 0.148 \\
$\begin{array}{l}\text { Variance of elevation of } \vec{u} \\
\text { (SD), degrees }\end{array}$ & $1063(381)$ & $1340(421)$ & 0.003 \\
\hline
\end{tabular}

Here, $\left[\omega_{x}, \omega_{y}, \omega_{z}\right]$ and $\vec{u}$ represent the angular velocity and rotation axis respectively.

The mean of angular velocity as well as the mean of the AzR and ElR did not reveal any significant statistical difference between men and women in this study. The variance of the ElR was significantly higher in men compared to women $(1340 \pm 421$ vs. $1063 \pm 381, \mathrm{P}=0.003$, respectively) as shown in table 2 .

Furthermore, we measured the correlation between demographics and our developed orientation parameters and reported the results in table 3 . The variance of AzR was significantly negatively correlated with height $(C C=-$ $0.26, \mathrm{P}=0.019$ ) while there was a significant positive correlation between the ElR and height ( $\mathrm{CC}=0.24$, $\mathrm{P}=0.034)$. Additionally, the ElR had a significant positive correlation with weight and BSA (CC $=0.22, \mathrm{P}=0.045$ and $\mathrm{CC}=0.26, \mathrm{P}=0.020$ respectively).

Figures 1 and 2 illustrates the AzR and ElR of $\overrightarrow{Q R S}$ vectors of two subjects together with their VCG QRS loop. The red, green and blue bars of the AzR (figures.1A \& 2A) and ElR (figures.1B \& 2B) plots correspond to their identical coloured QRS vectors on the VCG QRS loops (figure.1C \& 2C). The figures were colour coded to represent the progress of timing sequence with red representing the beginning. As it can be seen, the AzR in the first subject (figure.1A), whose VCG QRS loop can be described with one single plane (figure.1C), changes more rapidly in the beginning and at the end of the QRS loop while it stays approximately constant in between. On the other hand, the AzR of the second subject (figure.2A), whose QRS loop is obtained by the intersection of multiple planes (figure.2C), alternate between -200 and +200 degrees throughout the QRS loop. Furthermore, the EIR stay approximately constant about 100 degrees in the first subject (figures.1B), while the ElR vary from 0 to 200 degrees in the second case (figures.2B).

Table 3. Correlation between demographics and orientation parameters

\begin{tabular}{|c|c|c|c|c|c|}
\hline \multicolumn{2}{|c|}{ Measurement } & BMI & Height & Weight & BSA \\
\hline \multirow{2}{*}{$\begin{array}{l}\text { Mean of } \\
\text { azimuth } \\
\text { of } \vec{u}\end{array}$} & CC & -0.001 & -0.11 & -0.05 & -0.08 \\
\hline & PV & 0.994 & 0.315 & 0.666 & 0.502 \\
\hline \multirow{2}{*}{$\begin{array}{l}\text { Var of } \\
\text { azimuth } \\
\text { of } \vec{u}\end{array}$} & CC & -0.03 & -0.26 & -0.17 & -0.21 \\
\hline & PV & 0.769 & 0.019 & 0.140 & 0.063 \\
\hline \multirow{2}{*}{$\begin{array}{l}\text { Mean } \\
\text { Elevation } \\
\text { of } \vec{u}\end{array}$} & CC & 0.03 & 0.15 & 0.12 & 0.14 \\
\hline & PV & 0.775 & 0.173 & 0.278 & 0.206 \\
\hline \multirow{2}{*}{$\begin{array}{l}\text { Var of } \\
\text { elevation } \\
\text { of } \vec{u}\end{array}$} & $\mathrm{CC}$ & 0.08 & 0.24 & 0.22 & 0.26 \\
\hline & PV & 0.474 & 0.034 & 0.045 & 0.020 \\
\hline
\end{tabular}

\section{Discussion}

In this paper, we studied the variation of orientation of the QRS vector and its frame of reference in the QRS loop of the VCG of healthy individuals using quaternions and Euler angles. We observed a significant increase in variance of the ElR in males compared to females. Furthermore, we found that the variance of ElR was positively correlated with height, weight and consequently the BSA. In addition, the variance of AzR showed a negative correlation with height.

In 1957, Milnor demonstrated that the folding phenomenon was observed in patients with heart disease [1]. Recently, using dihedral angles we observed that the similar folding phenomenon was a predominant characteristic of QRS loop in patients with ventricular tachycardia [2]. Accordingly, to better characterize the folding phenomenon and study its underlying mechanisms and association with clinical characteristics, we presented a novel improved methodology in this paper to track the changes in the orientation of QRS vectors and the rotation of their frames of reference from sample to sample. Interestingly, in this study on healthy individuals we observed the folding phenomenon in 40 subjects (23 males), visually characterized by GS. This suggests that underlying physiological mechanisms other than cardiac abnormalities are responsible for folding. In addition, the increase in variance of ElR in males might be explained by the higher number of folding phenomenon observed in 
males compared to females. Therefore, the variance of ElR can be used to characterize folding.

However, the observation of folding phenomenon in healthy males and females with no history of any chronic disease is interesting and requires further analyses. In future study we plan to further develop quantitative parameters to precisely measure and capture the folding phenomenon and study them under various clinical settings to understand its underlying physiological mechanism.
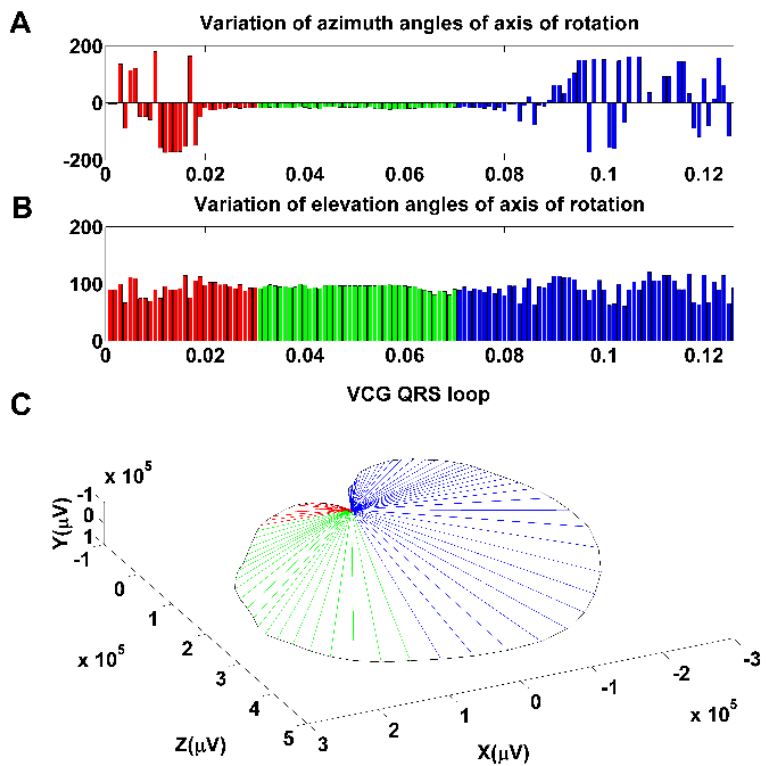

Figure 1. Plots of the (A) azimuth and (B) elevation of the rotation axis of $\overrightarrow{\mathrm{QRS}}$ respectively in first subject. (C) VCG QRS loop. The red, green and blue segments represent the progress of timing sequence with red representing the beginning.

A

A
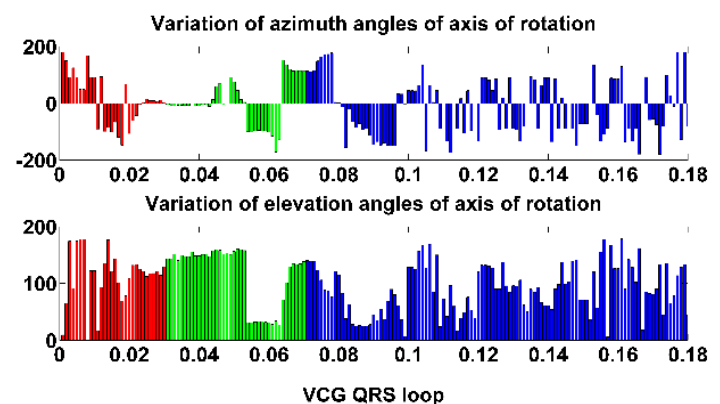

C

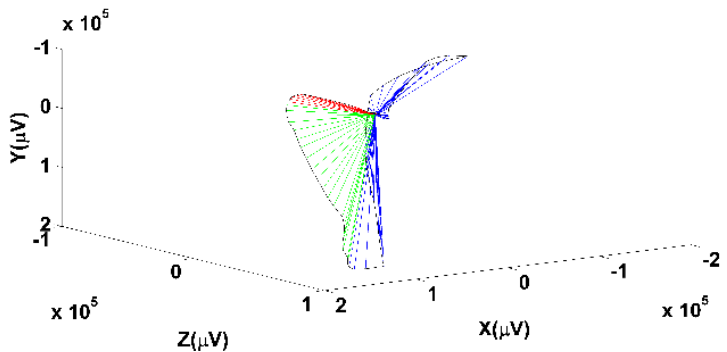

Figure 2. Plots of the (A) azimuth and (B) elevation of the rotation axis of $\overrightarrow{\mathrm{QRS}}$ respectively in second subject. (C) VCG QRS loop.

\section{Conclusion}

Study of variation of orientation and rotation of $\overrightarrow{\mathrm{QRS}}$ vectors from sample to sample can add important information to the already existing VCG parameters as well as help us to better characterize the folding phenomenon and its underlying mechanisms. Prospective study is needed to validate the clinical importance of the parameters developed in this paper.

\section{Acknowledgements}

We would like to show our gratitude to the National Institute of Health \#1R01HL118277 for its support.

\section{References}

[1] Milnor WR. The normal vectorcardiogram and a system for the classification of vectorcardiographic abnormalities. Circulation 1957;16:95-106.

[2] Sedaghat G, Ghafoori E, Waks JW, Kabir MM, Shvilkin A, Josephson ME, Tereshchenko LG. Quantitative Assessment of Vectorcardiographic Loop Morphology. J Electrocardiol 2016;49:154-63.

[3] Couderc JP, Xiaojuan X, Zareba W, Moss AJ. Assessment of the stability of the individual-based correction of QT interval for heart rate. Ann Noninvasive Electrocardiol 2005;10:25-34.

[4] Sur S, Han L, Tereshchenko LG. Comparison of sum absolute QRST integral, and temporal variability in depolarization and repolarization, measured by dynamic vectorcardiography approach, in healthy men and women. PLoS One 2013;8:e57175.

[5] Waks JW, Soliman EZ, Henrikson CA, Sotoodehnia N, Han L, Agarwal SK, et al. Beat-to-beat spatiotemporal variability in the $\mathrm{T}$ vector is associated with sudden cardiac death in participants without left ventricular hypertrophy: the Atherosclerosis Risk in Communities (ARIC) Study. J Am Heart Assoc 2015;4:e001357.

[6] Diebel, J.: Representing attitude: Euler angles, unit Quaternions, and rotation vectors. Tech. rep., Stanford University,California, USA (2006)

[7] Kuipers JB, Quaternions and Rotation Sequences: Princeton University Press, 1999.

[8] Goldstein H, The Euler Angles and Euler Angles in Alternate Conventions. and Appendix B in Classical Mechanics, 2nd ed: Reading, MA: Addison-Wesley 1980;4:143-148 and 606-610

[9] Landau LD, Lifschitz EM, Mechanics: Oxford England: Pergamon Press 1976; 3rd ed.

Address for correspondence.

Golriz Sedaghat.

Knight Cardiovascular Institute, Oregon Health and Science University, Portland, OR 97239, USA.

E-mail address: sedaghat@ohsu.edu 